\title{
Los comités de investigación en salud: su autoridad, responsabilidad fundamental y necesidad de que se sometan a auditorías periódicas
}

Edith Valdez-Martínez ${ }^{*}$ y Miguel Bedolla²

${ }^{1}$ Instituto Mexicano del Seguro Social, Coordinación de Investigación en Salud, Ciudad de México, México; ${ }^{2}$ Universidad de Texas, Centro de Estudios de Políticas Públicas, Texas, Estados Unidos de América

\section{Resumen}

La ética de la investigación debe incluir las dimensiones teórica y práctica. La primera la conforman regulaciones y directrices y la segunda alude a la forma cómo los comités de ética y ética de la investigación interpretan y aplican esas regulaciones y directrices. En este artículo se analiza la operación de los comités a nivel práctico. Dado que la evaluación y el dictamen de los protocolos de investigación es un proceso que requiere consciencia plena, la omisión de esta conlleva importantes implicaciones para la investigación en salud.

PALABRAS CLAVE: Comités de ética. Ética. Bioética. Auditoría. Investigación médica.

\section{Abstract}

Research ethics must include theoretical and practical dimensions. The first one is structured by regulations and policies, and the second dimension refers to how the committee interprets and applies those regulations and policies. This article analyses the operation of the committees at a practical level. Given that the evaluation and judgement of research protocols is a process that requires full awareness, its omission entails important implications for health research.

KEY WORDS: Ethics committees. Ethics. Bioethics Audit. Medical research.

\section{Introducción}

La ética es el estudio de la conducta de los humanos en relación con la responsabilidad que tienen de elegir (cuando una elección es posible) lo que es bueno o mejor u óptimo para la construcción de un bien común verdaderamente bueno para nuestra sociedad. Ese bien común se construye a través de elecciones-decisiones basadas en valores como la solidaridad, la responsabilidad y la confianza. ${ }^{1}$ La palabra responsabilidad significa elegir la opción que es buena, mejor u óptima porque está de acuerdo con los principios que nos interpelen espontáneamente; y esto es así por el hecho de estar conscientes de que somos conocedores de las condiciones en las que vivimos y los protagonistas que actúan en la sociedad que creamos juntos.

Consecuentemente, la responsabilidad implica que estamos en una situación en la que tenemos dos o
Gac Med Mex. 2019;155:406-409 Disponible en PubMed www.gacetamedicademexico.com 
más opciones y que hay otras personas cuyo bien debemos tomar en cuenta al hacer nuestra elección. Esas otras personas reclaman de forma legítima que la opción elegida sea también la buena, mejor u óptima para todos. ${ }^{2}$ Para ayudarnos a elegir, en los comités que nos ocupa, tenemos un conjunto de regulaciones y directrices de aceptación internacional y nacional. Regulaciones y directrices derivadas de la convención y voluntad humanas, y aceptadas después de haber sido analizadas racionalmente y de decidir que son buenas para el bien individual y común, y que sirven para guiar el trabajo de los comités.

En México, la evaluación de los protocolos de investigación que involucran humanos como sujetos de investigación se hace a través de grupos independientes: los comités de investigación $(\mathrm{Cl})^{3}$ y los comités de ética de la investigación (CEI). ${ }^{4}$ En su trabajo de revisión de protocolos, gran parte sus responsabilidades son las mismas. Los Cl evalúan y dictaminan que el protocolo de una investigación tenga un objetivo científico claro, que la metodología propuesta tenga el poder suficiente para alcanzar el objetivo del estudio, que el plan de análisis de datos sea apropiado para la naturaleza de las variables y los objetivos, además de evaluar la importancia social, científica y clínica del protocolo. ${ }^{3}$ En tanto, los CEI evalúan y dictaminan, desde el punto de vista ético, los propósitos, objetivos y métodos que se presentan en el protocolo de investigación. ${ }^{5}$ Además de estos dos comités, otro funciona independientemente: el comité de bioseguridad. Este último revisa y analiza los protocolos de investigación que implican técnicas de ingeniería genética, uso de isotopos radiactivos y dispositivos generadores de radiaciones ionizantes o electromagnéticas. ${ }^{3}$

El trabajo de los comités es indiscutiblemente importante y necesario porque, teóricamente, asegura que las propuestas clínicas de investigación sean éticas. ${ }^{6}$ Sin embargo, no podemos ignorar que en la práctica de los comités aún existen temas que deben ser resueltos, por ejemplo, algunos investigadores argumentan que los comités no se responsabilizan de las decisiones y sugerencias que emiten, otros señalan que son frecuentes las decisiones injustificadas 0 inadecuadamente justificadas, y unos más argumentan que los comités rechazan los protocolo de investigación de enfoque exploratorio (cuando la revisión de la literatura arrojó elementos insuficientes para establecer una hipótesis a priori ${ }^{7}$ ) o de enfoque inductivo (por el cual se obtiene la hipótesis a posteriori, esto es, a partir de la observación de casos particulares ${ }^{7}$ ) por carecer de hipótesis.

Hay quienes señalan que los comités han demostrado ser incapaces de entender y evaluar adecuadamente las investigaciones que plantean metodologías científicas "novedosas" o modernas. La consecuencia de que los comités operen bajo la sombra de la ignorancia e incompetencia tiene un claro costo social, porque al rechazar protocolos de investigación con validez científica y con importancia social, científica y clínica, se retrasan o impiden mejoras en la salud y el bienestar de la población; más precisamente, retrasan o impiden el mejoramiento del bien individual y colectivo de los servicios de salud.

\section{La dimensión regulatoria}

El ámbito regulatorio lo conforman las guías éticas internacionales (por ejemplo, el Reporte Belmont, la Declaración de Helsinki, las normas del Consejo de Organizaciones Internacionales de las Ciencias Médicas, la Declaración Internacional sobre Datos Genéticos Humanos, etcétera), la normatividad nacional propia de cada país y la normatividad propia de cada institución o establecimiento donde se realiza investigación para la salud. Estas dos últimas deben ser congruentes con las disposiciones internacionales. La dimensión regulatoria deriva de la convención y las voluntades humanas, sus propósitos son:

- Proteger los derechos e intereses de los sujetos de investigación.

- Brindar un marco de análisis que guíe a los miembros de los $\mathrm{Cl}$ y $\mathrm{CEI}$ en la revisión y evaluación de los protocolos.

En México, existen guías nacionales para la integración y funcionamiento de los $\mathrm{Cl}^{3}$ y CEI, ${ }^{5}$ las cuales están alineadas con las guías operacionales emitidas por la Organización Mundial de la Salud. ${ }^{8,9}$ Son un conjunto de directivas objetivas que especifican cómo deben integrarse y funcionar los $\mathrm{Cl}$ y $\mathrm{CEI}$, por ejemplo, respecto al último, entre sus atribuciones se estipulan las siguientes: ${ }^{5}$

- "Asesorar a los titulares de los establecimientos e instituciones para la emisión de dictámenes... con respecto a la aprobación de investigaciones en sus unidades de responsabilidad".

- "Coadyuvar en la aplicación de la Ley, el Reglamento y las demás disposiciones aplicables en materia de investigación en salud".

- "Contribuir a salvaguardar la dignidad, los derechos, la seguridad y el bienestar de todos los 
actuales o potenciales participantes en las investigaciones".

De acuerdo con estas directivas, la operación de Ios CEI constituye el papel central de su trabajo; por medio de ellas, los CEI ejercen poder y autoridad moral para evaluar y dictaminar sobre la investigación en salud.

Quienes presiden e integran los $\mathrm{Cl}$ y los CEI deben entender que el ámbito regulatorio (códigos, declaraciones, normas), aunque indispensable, no es exhaustivo. Solo para ilustrar: ninguna de las regulaciones mencionadas considera las implicaciones de los valores de orden (aquellos que crean y mantienen y hacen progresar el bien común), como la responsabilidad, reciprocidad y alianza social. Si los comités hicieran suyos estos valores, su operación en el ámbito regulatorio contribuiría al bien común, expresado como la generación de conocimiento válido con la potencialidad de producir beneficios sociales sustanciales.

Aunque los miembros de los $\mathrm{Cl}$ y $\mathrm{CEI}$ operen bajo un sistema de reglas, requieren una deliberación adecuadamente contextualizada, ${ }^{1}$ que implica conocimiento de las regulaciones, entendimiento de los principios éticos, familiarización con las fortalezas y debilidades de los diferentes diseños de estudio y conocer o interesarse por conocer el tema del protocolo que evalúan. Todo ello con el propósito de verdaderamente comprender lo que se plantea en el protocolo, para que, de esta forma, la operación de los comités sea racional y responsable y su autoridad sea ejercida legítimamente al emitir un dictamen. De lo contrario, la práctica de los comités será rígida, injusta, irracional e irresponsable, características propias de un sistema de fuerza y arbitrariedad. Esto se hace evidente cuando los miembros de los comités malinterpretan lo que los autores han declarado en su protocolo, cuando el proceso de deliberación es influido por intereses personales, cuando sus juicios están guiados por la proyección de las experiencias o expectativas personales y cuando se lleva a cabo una revisión estandarizada para todos los protocolos, aunque estos presenten diferentes enfoques metodológicos.

Ante la ausencia de racionalidad y responsabilidad, la autoridad de los comités será ilegítima y no tiene por qué ser obedecida por quienes enviaron protocolos de investigación para su dictamen. De hecho, esos investigadores tienen la obligación de oponerse prudentemente a que sigan funcionando los comités que no operan racional y responsablemente.

\section{La dimensión práctica}

La dimensión práctica a la que hacemos referencia tiene que ver con el ethos o carácter habitual de una persona; este incluye la forma de pensar y ser, a la luz de las emociones que siente el individuo, de las expectativas, valores e intereses que lo motivan y la manera como influyen en sus juicios y decisiones. ${ }^{1}$ De ahí que las regulaciones y directrices que guían el trabajo de los comités sean entendidas y cumplidas de forma diferente, tanto por cada uno de sus miembros como por el comité en su conjunto. Por eso, los miembros de los $\mathrm{Cl}$ y $\mathrm{CEI}$ deben hacer un esfuerzo constante por educar sus emociones, sensaciones y pensamientos, y armonizarlos con las expectativas de racionalidad y responsabilidad con las que les ha sido delegada la autoridad para emitir juicios y dictámenes. Se requiere que cada miembro de un comité despliegue la estructura formalmente dinámica de la consciencia humana, con el propósito de lograr juicios verdaderamente éticos y no juicios técnicos, de conveniencia o utilidad que dañen los intereses de las personas que serán afectadas por lo que decida el comité.

Para ilustrar la importancia de la dimensión práctica, un ejemplo: aun cuando existen rigurosas normas de construcción y estándares de arquitectura, que el Estado ha emitido para la construcción de edificios, varios inmuebles nuevos se derrumbaron en México con los terremotos acontecidos en los últimos años. Las estructuras arquitectónicas de esos edificios derrumbados son testimonio de la dimensión práctica, del carácter o forma de pensar y ser de quienes los diseñaron y construyeron: arquitectos, ingenieros, albañiles. Extrapolando el ejemplo al trabajo de los comités, estos en su operación no pueden ser simples seguidores de normas objetivas, porque al hacerlo socaban o debilitan la credibilidad en la ciencia.

Un rasgo característico atribuido a los comités es su "autonomía", aunque en la vida real su conformación y funcionamiento están directamente vinculados a la autoridad de la institución a la que sirven. ${ }^{3,5}$ Debe advertirse que su autonomía está dada y claramente delimitada por las guías y los lineamientos del ámbito en el que funcionan. La autonomía no significa que los comités puedan hacer lo que deseen o lo que se les ocurra, de ser así sus actos serían arbitrarios y su operación ilegítima. La autonomía de los comités es distinta de la autonomía de las personas que deciden, voluntariamente previa información, participar en una investigación. 
Está demostrado que implementar un sistema de auditoría para examinar el trabajo de los comités ayudaría, no a enjuiciar a los comités y sus miembros, sino a descubrir áreas de cambio y mejora. ${ }^{10}$

\section{Conclusión}

Actualmente, los $\mathrm{Cl}$ y $\mathrm{CEI}$ tienen un papel central y gran poder y autoridad sobre la investigación en salud, por lo que es importante que operen clara y transparentemente. El proceso de revisión de los protocolos de investigación es un asunto serio que debe ser conducido justamente. Se trata de que los miembros de los comités cumplan cabalmente con su responsabilidad personal y los comités como entidad cumplan con su responsabilidad social, de ahí la imperiosa necesidad de evaluar regularmente a los $\mathrm{Cl}$ y CEI a través de auditorías.

\section{Bibliografía}

1. Beauchamp TL, Childress JF. Principles of biomedical ethics. EE. UU.: Oxford University Press; 2013.

2. Gómez-De Silva G. Breve diccionario etimológico de la lengua española. México: Fondo de Cultura Económica; 1998.

3. Norma Oficial Mexicana NOM-012-SSA3-2012, que establece los criterios para la ejecución de proyectos de investigación en salud en seres humanos. México: Diario Oficial de la Federación 2013 Ene 4.

4. Decreto por el que se adiciona el Artículo 41 Bis y se reforma el Artículo 98 de la Ley General de Salud. México: Diario Oficial de la Federación 2011 Dic 14.

5. Secretaría de Salud. Guía Nacional para la Integración y el Funcionamiento de los Comités de Ética en Investigación. México: Comisión Nacional de Bioética/Secretaría de Salud; 2018.

6. Declaración de Helsinki. Finlandia: Asociación Médica Mundial; 2013.

7. Hernández-Sampieri R, Fernández-Collado C, Baptista-Lucio P. Metodología de la investigación. México: McGraw Hill; 2003.

8. Organización Mundial de la Salud. Estándares y guías operacionales para la revisión ética de investigación en salud con participantes humanos. Suiza: Organización Mundial de la Salud; 2011.

9. Organización Mundial de la Salud. Guías operacionales para comités de ética que evalúan investigación biomédica. Suiza: Organización Mundial de la Salud; 2000.

10. Valdez-Martínez E, Trumbull B, Garduño-Espinosa J, Porter J. Understanding the structure and practices of research ethics committees through research and audit: a study from Mexico. Health Policy. 2005;74:56-68. 